\title{
Matter-wave two-dimensional solitons in crossed linear and nonlinear optical lattices
}

\author{
H. L. F. da Luz, ${ }^{1}$ F. Kh. Abdullaev, ${ }^{2}$ A. Gammal,${ }^{1}$ M. Salerno, ${ }^{3}$ and Lauro Tomio ${ }^{4,5}$ \\ ${ }^{1}$ Instituto de Física, Universidade de São Paulo, 05508-090 São Paulo, São Paulo, Brazil \\ ${ }^{2}$ CFTC, Complexo Interdisciplinar, Universidade Lisboa, Avenida Professor Gama Pinto, 2, P-1649-003 Lisboa, Portugal \\ ${ }^{3}$ Dipartimento di Fisica "E.R. Caianiello", CNISM and INFN-Gruppo Collegato di Salerno, Università di Salerno, \\ Via Ponte don Melillo, I-84084 Fisciano (SA), Italy \\ ${ }^{4}$ Instituto de Física, Universidade Federal Fluminense, 24210-346 Niterói, Rio de Janeiro, Brazil \\ ${ }^{5}$ Instituto de Física Teórica, Universidade Estadual Paulista (UNESP), 01140-070 São Paulo, São Paulo, Brazil
}

(Received 25 August 2010; published 22 October 2010)

\begin{abstract}
The existence of multidimensional matter-wave solitons in a crossed optical lattice (OL) with a linear optical lattice (LOL) in the $x$ direction and a nonlinear optical lattice (NOL) in the $y$ direction, where the NOL can be generated by a periodic spatial modulation of the scattering length using an optically induced Feshbach resonance is demonstrated. In particular, we show that such crossed LOLs and NOLs allow for stabilizing two-dimensional solitons against decay or collapse for both attractive and repulsive interactions. The solutions for the soliton stability are investigated analytically, by using a multi-Gaussian variational approach, with the Vakhitov-Kolokolov necessary criterion for stability; and numerically, by using the relaxation method and direct numerical time integrations of the Gross-Pitaevskii equation. Very good agreement of the results corresponding to both treatments is observed.
\end{abstract}

DOI: 10.1103/PhysRevA.82.043618

PACS number(s): 67.85.Hj, 03.75.Lm, 67.85.Jk, 03.75.Kk

\section{INTRODUCTION}

Bose-Einstein condensates (BECs) in optical lattices (OLs) are presently attracting a great deal of interest, both experimentally and theoretically [1,2], in connection with a series of physical phenomena, which arise in condensed-matter physics; these include Bloch oscillations [3-5], generation of coherent atomic pulses (atom laser) [6], dynamical localization [7-9], Landau-Zener tunneling [10-12], superfluid Mott transitions [13], etc. The flexibility of BEC systems with respect to changes of parameters indeed permits the investigation of these phenomena in a much easier way than in their condensedmatter physics counterparts. On the other hand, BEC systems are intrinsically nonlinear, and the correspondence with usual condensed-matter phenomena can be established mainly in the linear regime when the interatomic interactions are detuned to zero by means of external magnetic fields using the Feshbach resonance (FR) technique [14]. The presence of nonlinearity, however, represents an additional resource for BEC systems, which leads to interesting phenomena, such as the existence of localized bound states, which remain stable for an infinite time due to a perfect balance between nonlinearity and dispersion. In the presence of a periodic potential or a linear optical lattice (LOL), these states are known as gap solitons (they have chemical potentials located inside the band gaps of the underlying linear band structure), which can exist for both attractive and repulsive interactions [15-17], this last fact only being possible due to the presence of the periodic potential. In this context, it has been shown that gap solitons are formed through the mechanism of modulational instability of the Bloch states, at the edges of the Brillouin zone of the underlying linear periodic system [18]. Their existence in BECs with repulsive interactions was experimentally demonstrated in Ref. [19]. Recently, the possibility of Bloch oscillations [5], Rabi oscillations [20], and dynamical localization [8] of BEC gap solitons, in the presence of constant or time-dependent linear forces (induced by accelerations of the OL), have also been considered. In these cases, besides the LOL, periodic spatial variations of the nonlinearity, also known as a nonlinear optical lattice (NOL), have been used in order to avoid dynamical instabilities and to make the foregoing phenomena long lived in the nonlinear regime.

In higher dimensions, LOLs were shown to be effective in stabilizing localized states against collapse or decay, leading to the formation of stable multidimensional gap solitons [21,22]. In particular, it has been shown that a one-dimensional (1D) LOL in three-dimensional (3D) space does not allow stabilizing 3D gap solitons. This soliton stabilization only becomes possible for attractive 3D BECs subjected to the action of a two-dimensional (2D) LOL [22,23]. The possibility for stabilizing solitons by means of NOL has been investigated mainly in the $1 \mathrm{D}$ case, for which the mathematical properties have been studied in detail, for the ground state when considering solitons supported by 1D NOL [24-27], as well as by combinations of LOLs and NOLs [28]. In the 2D case, it was shown in Ref. [29] that, for conservative systems, an NOL in one direction by itself cannot give stable localized solutions in the case of attractive interactions. This fact also remains true for 2D NOLs both for attractive and repulsive interactions.

Since NOLs can be created by means of external magnetic fields via the usual FR technique, or by optically induced Feshbach resonances (OFRs) [30], it is of interest to investigate whether crossed combined LOLs and NOLs (e.g., an LOL in one direction plus an NOL in the orthogonal direction) allow for stabilizing multidimensional solitons. This analysis can be relevant in the perspective of experimental observations of multidimensional solitons. In this regard, we remark that an experimental realization of an NOL was recently achieved (on the submicrometer scale) for a ${ }^{174} \mathrm{Yb}$ BEC using the OFR technique [31]. Moreover, we recall that, to date, no multidimensional BEC solitons in LOLs have been observed. Besides, offering the possibility to explore alternate methods 
for multidimensional soliton creation, crossed combined LOLs and NOLs can allow extending a 2D setting interesting transmission and reflection phenomena of soliton wave packets, such as matter-wave optical limiting processes and bistability phenomena considered in the $1 \mathrm{D}$ case for potential applications as matter-wave limiters, BEC mirrors or cavities, and atomic switches [32-34].

The aim of the present paper is to investigate the existence of 2D matter-wave solitons in crossed OLs consisting of an LOL in the $x$ direction and an NOL in the $y$ direction. In particular, we show that crossed LOLs and NOLs allow stabilizing 2D solitons both for attractive and for repulsive interatomic interactions. This will be demonstrated by means of analytical and numerical approaches: analytically, with the variational method and a multi-Gaussian ansatz; numerically, with relaxation methods and direct numerical integrations of the Gross-Pitaevskii (GP) differential equation. Due to the lattice anisotropy, the solitons display elliptical cross sections, which, in our variational approach (VA), are accounted for by a multi-Gaussian ansatz with different parameters for each spatial direction. Existent VA curves and corresponding stability properties of multidimensional solitons are given for several parameter values in terms of chemical potentials and total energies as functions of the number of particles, using the Vakhitov-Kolokolov (VK) criterion for the stability. The results are then compared with direct numerical integrations of the full Gross-Pitaevskii equation (GPE).

The paper is structured as follows. In Sec. II, we introduce the model equations and discuss the physical implementation of a 2D crossed LOL and NOL by using spatial modulations of the scattering length. In Sec. III, we use a multi-Gaussian VA to derive our results, presented for chemical potentials and total energies as functions of the number of atoms, for the case of 2D solitons in 2D crossed OLs, for both attractive and repulsive interactions, where the stability is investigated by the well-known VK criterion. In Sec. IV, the results of the VA are compared with the ones obtained by direct integrations of the GPE, using both relaxation in imaginary time and real time propagations to check the stability. Finally, in Sec. V, the main results of the paper are briefly summarized.

\section{MODEL EQUATIONS}

Multidimensional BECs in 2D crossed LOLs and NOLs are described in the mean-field approximation by the following GPE:

$$
i \hbar \frac{\partial \psi}{\partial t}=-\frac{\hbar^{2}}{2 m} \nabla^{2} \psi-\Lambda \cos (2 k x) \psi-g(y)|\psi|^{2} \psi,
$$

where $\psi \equiv \psi(\mathbf{r}, t)$ is normalized to the number of atoms, $\nabla^{2}$ denotes the Laplacian in $2 \mathrm{D}, m$ is the atomic mass, and $\Lambda \cos (2 k x)$ is an LOL in the $x$ direction, with strength $\Lambda$ and lattice constant $\pi / k$. The function $g(y)$ represents an NOL in the $y$ direction, produced either by spatially varying magnetic fields near an FR or by OFRs [30]. It the following, we assume $g(y)$ of the form

$$
g(y)=g_{0}+g_{1} \cos (2 \kappa y),
$$

where $g_{0}$ denotes the mean nonlinearity related to the mean $s$-wave scattering length $a_{s 0}$, and $g_{1}$ is the strength of a periodic modulation of the nonlinearity in the $y$ direction, having the period $\pi / \kappa$. Note that the quasi-2D system is confined in the $z$ direction, with the effective 2D scattering length being given in terms of the $3 \mathrm{D}$ scattering length and some typical scale in the confined direction [35,36]. In this case, the parameters $g_{0}$ and $g_{1}$ are given in units of energy multiplied by some squared distance. The spatial modulation can be produced by manipulating the scattering length with a laser field tuned near a photoassociation transition (e.g., close to the resonance of one of the bound $p$ levels of the excited molecules). Virtual radiative transitions of a pair of interacting atoms to this level can change the value and even reverse the sign of the scattering length. One can show that a periodic variation of the laser field intensity applied in the $y$ direction of the form $I(y)=I_{0} \cos ^{2}(\kappa y)$ produces a periodic variation of the atomic scattering length such that $a_{s}(y)=a_{s 0}[1+\alpha I /(\delta+I)]$, where $a_{s 0}$ is the scattering length in the absence of light, $\delta$ is the frequency detuning of the light from the resonance, and $\alpha$ is a constant factor [24,30]. For weak intensities, when $I_{0} \ll|\delta|$, we have that the real part of the scattering length can be approximated by $a_{s}=a_{s 0}+a_{s 1} \cos ^{2}(\kappa y)$, leading to a modulated nonlinearity of essentially the same form assumed in Eq. (2).

It should be remarked, however, that the creation of an NOL in a BEC, by manipulation of the scattering length in this manner, also implies some spontaneous emission loss, which is inherent in the OFR technique [37-39]. Such dissipative effects can be strongly reduced by using laser fields with sufficiently high intensity and detuned from the resonance [40]. It is also worth pointing out that, by using a laser field to control a magnetic FR, the losses can also essentially be reduced in comparison with OFR. In particular, the experiment reported in Ref. [40] demonstrates that a laser light, near the resonance with a molecular bound-to-bound transition in ${ }^{87} \mathrm{Rb}$, can be used to shift the value of the magnetic field where the FR occurs. In this way, it is possible to vary the scattering length on the optical wavelength scale, without having considerable losses (about 2 orders of magnitude lower than in OFR experiments). We also remark that periodic variations of the scattering length, induced by the usual FR technique using spatially periodic external magnetic fields, would lead to similar conclusions.

In the following, we adopt dimensionless units by rescaling the space and time variables such that the variables in Eq. (1) are redefined according to $t \rightarrow\left(\hbar / E_{r}\right) t, \mathbf{r} \rightarrow \mathbf{r} / k$, with $E_{r} \equiv$ $\hbar^{2} k^{2} / 2 m$ being the recoil energy and $\mathbf{r} \equiv\{x, y, z\}$. The wave function is rescaled as $\psi(\mathbf{r}, t) \rightarrow k u(\mathbf{r}, t)$, in terms of which Eq. (1) acquires the form

$$
i \frac{\partial u}{\partial t}=-\left[\frac{\partial^{2} u}{\partial x^{2}}+\frac{\partial^{2} u}{\partial y^{2}}\right]-V_{l}(x) u-\Gamma(y)|u|^{2} u,
$$

where

$$
V_{l}(x)=\varepsilon \cos (2 x), \quad \Gamma(y)=\chi+\gamma \cos (\lambda y)
$$

denote the LOL and NOL interactions, respectively. In the foregoing, $\varepsilon=\Lambda / E_{r}, \quad \chi=g_{0} k^{2} / E_{r}=8 \pi a_{s 0} k, \quad \lambda=2 \kappa / k$, and $\gamma=g_{1} k^{2} / E_{r}$, with $g_{1}$ assumed as a free parameter. 


\section{VARIATIONAL ANALYSIS}

We consider localized solutions of Eq. (3) in the crossed LOLs and NOLs given by Eq. (4), in the cases that the mean nonlinearity $\chi$ can be both negative (repulsive interaction) and positive (attractive interaction). In order to obtain analytical estimates for the soliton existence and stability, we use the VA and look for solutions of the form

$$
u(x, y, t)=U(x, y) \exp (-i \mu t),
$$

where $\mu$ is the chemical potential and $U(x, y)$ is a real function for the soliton profile. Due to the anisotropy of the crossed OL, having different natures and different strengths in the two directions, we expect elliptical cross sections for the soliton profiles. This feature can be accounted for in our analysis of Eq. (5) by adopting the following ansatz for $U(x, y)$ :

$$
U(x, y)=A e^{-\left(a x^{2}+b y^{2}\right) / 2},
$$

where $a$ and $b$ are the parameters controlling the Gaussian widths in the two directions, with $a / b$ as the aspect ratio of the solution. Using this ansatz, the stationary GPE can be written as

$$
\mu U+\frac{\partial^{2} U}{\partial x^{2}}+\frac{\partial^{2} U}{\partial y^{2}}+\varepsilon \cos (2 x) U+[\chi+\gamma \cos (\lambda y)] U^{3}=0
$$

from which the chemical potential and corresponding total energy, respectively, are given by

$$
\begin{aligned}
& \mu N=\int\left\{|\nabla U|^{2}-\varepsilon \cos (2 x) U^{2}-[\chi+\gamma \cos (\lambda y)] U^{4}\right\} d x d y, \\
& E=\int\left\{|\nabla U|^{2}-\varepsilon \cos (2 x) U^{2}-\frac{\chi+\gamma \cos (\lambda y)}{2} U^{4}\right\} d x d y .
\end{aligned}
$$

The integrations in the preceding and following expressions cover the $2 \mathrm{D}$ phase space, from $-\infty$ to $+\infty$. From the ansatz (6), it follows that the normalized number of atoms is expressed in terms of the variational parameters $A, a$, and $b$, as

$$
N=\int U^{2} d x d y=\frac{A^{2} \pi}{\sqrt{a b}} .
$$

The stationary GPE in Eq. (7) can be derived from the following field Lagrangian:

$$
\begin{aligned}
L= & \frac{1}{2} \int\left\{|\nabla U|^{2}-[\mu+\varepsilon \cos (2 x)] U^{2}\right. \\
& \left.-\frac{1}{2}[\chi+\gamma \cos (\lambda y)] U^{4}\right\} d x d y .
\end{aligned}
$$

By substituting the ansatz (6) into the previous Lagrangian and performing the integrations, in terms of the variational parameters $a, b$, and $A$, we obtain the following effective Lagrangian:

$L_{\mathrm{eff}}=\frac{A^{2} \pi}{4 \sqrt{a b}}\left\{a+b-\frac{A^{2}}{2}\left[\chi+\gamma e^{-\lambda^{2} /(8 b)}\right]-2\left[\mu+\varepsilon e^{-1 / a}\right]\right\}$,

with the corresponding total energy given by

$$
E=N\left\{\frac{a+b}{2}-\frac{A^{2}}{4}\left[\chi+\gamma e^{-\lambda^{2} /(8 b)}\right]-\varepsilon e^{-1 / a}\right\} .
$$

Then, following from $L_{\text {eff }}$, by using the Euler-Lagrange equations for the parameters $a, b$, and $A$, we can derive the relations for the chemical potential, total energy, and number of atoms. One can easily show that these relations can be written as

$$
\begin{gathered}
\mu=\frac{b-3 a}{2}-\varepsilon\left(1-\frac{4}{a}\right) e^{-1 / a}, \\
N=\frac{4 \pi}{a \sqrt{a b}} \frac{a^{2}-2 \varepsilon e^{-1 / a}}{\chi+\gamma e^{-\lambda^{2} / 8 b},} \\
\frac{E}{N}=\frac{b-a}{2}-\varepsilon\left(1-\frac{2}{a}\right) e^{-1 / a},
\end{gathered}
$$

with $a, b$ solutions of the transcendental equation,

$$
\frac{1}{b}+\gamma \frac{\lambda^{2}}{4 b^{2}} \frac{e^{-\lambda^{2} / 8 b}}{\chi+\gamma e^{-\lambda^{2} / 8 b}}=F(a)
$$

where

$$
F(a)=\frac{a}{a^{2}-2 \varepsilon e^{-1 / a}} .
$$

Exact analytical solutions of Eq. (15) can be obtained in the following limit.

(i) Case $\gamma=0$. In this case, only the LOL in the $x$ direction is present, and from Eq. (15), we obtain $b=1 / F(a)$. By substituting this value of $b$ in Eqs. (12) and (13), we get the following parametric equations, in the $\mu-N$ space, for the existent curves of a soliton:

$$
\begin{array}{r}
\mu=-a-\varepsilon\left(1-\frac{3}{a}\right) e^{-1 / a}, \\
N=\frac{4 \pi}{\chi} \sqrt{1-\varepsilon \frac{2}{a^{2}} e^{-1 / a} .}
\end{array}
$$

Notice from Eq. (10), that this case becomes equivalent to the case with $\lambda=0$ and $\gamma \neq 0$, by replacing $\chi$ in Eq. (18) with $\chi+\gamma$. The preceding equations coincide with the ones of the 1D potential case investigated in Ref. [23] [compare the foregoing equations with Eqs. (8) and (9) of this paper], where it was shown that the system can support 2D localized solutions only for attractive interactions. The absence of a confining potential in the $y$ direction makes the solution uniform in this direction. But, interestingly enough, the solution is stable if the periodic boundary conditions are adopted in the $y$ direction (line soliton).

(ii) Case $\chi=0$. In this case, both the LOL and the NOL are present, but the mean nonlinearity is detuned to zero. Then, Eq. (15) reduces to $4 b+\lambda^{2}=4 b^{2} F(a)$, and $b$ can be expressed in terms of parameter $a$ as

$$
b=\frac{1+\sqrt{1+\lambda^{2} F(a)}}{2 F(a)} .
$$

(iii) Case $\varepsilon=0$. In this case, only the NOL in the $y$ direction is present, and from Eq. (15), we have that $F(a)=1 / a$. The solutions for $\mu$ and $N$ can be conveniently expressed in terms 

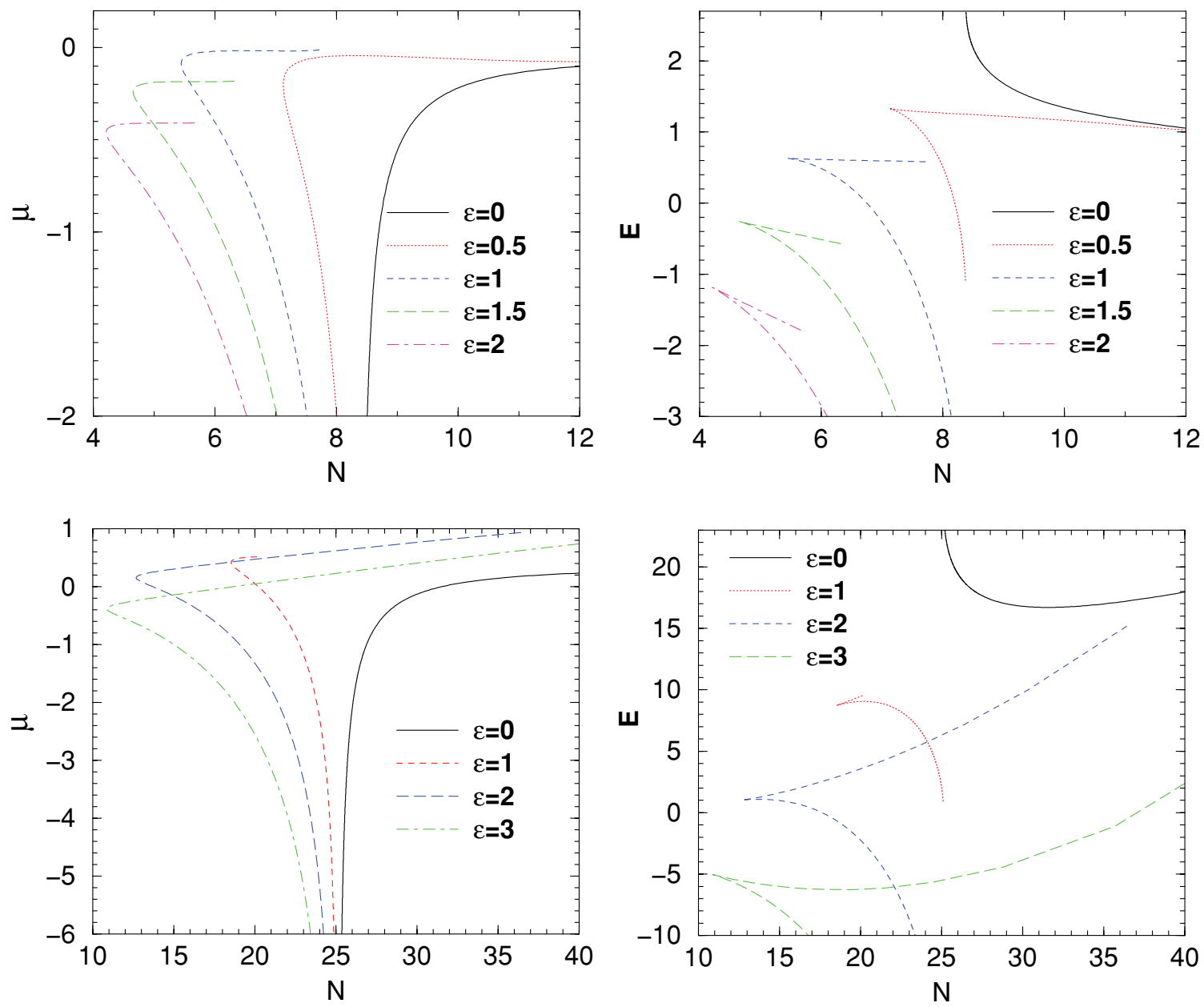

FIG. 1. (Color online) VA results for 2D solitons, for attractive (upper panels, with $\chi=0.5$ ) and repulsive (lower panels, with $\chi=-0.5$ ) mean nonlinearities. The chemical potentials $\mu$ are shown in the left panels, with the total energies $E$ shown in the right panels, as functions of $N$ for increasing strengths of the LOL $\varepsilon$, as indicated inside the panels. Other parameters are fixed as $\lambda=2, \gamma=1.0$. All the quantities are in dimensionless units.

of parameter $b$ as

$$
\begin{gathered}
\frac{a}{b}=\left[1+\frac{\lambda^{2}}{4 b}\left(1-\frac{\chi}{\chi+\gamma e^{-\lambda^{2} / 8 b}}\right)\right]^{-1}, \\
\mu=\frac{b}{2}\left(1-3 \frac{a}{b}\right), \\
N=4 \pi \sqrt{\frac{a}{b}} \frac{1}{\chi+\gamma e^{-\lambda^{2} / 8 b}} .
\end{gathered}
$$

In the absence of any confinement in the $x$ and $y$ directions (e.g., for $\varepsilon=0$ and $\gamma=0$ ), the previous equations reproduce the known results of the 2D nonlinear Schrödinger solitons. In particular, for attractive interactions $(\chi>0)$, the soliton widths in the two directions become equal $(a=b)$, and the number of atoms times the nonlinearity reduces to a constant, which coincides with the norm of 2D Townes solitons [41] determining the critical threshold for collapse of a soliton in $2 \mathrm{D}$ as $N_{\text {cr }}=4 \pi / \chi$.

In the general case, the solution of Eq. (15) must be found numerically. In Fig. 1, we show variational curves $N, \mu$ (left panels) obtained from the preceding equations for attractive (top) and repulsive (bottom) cases. Notice the nonmonotonic behavior with the appearance of a minimum (turning point) in $N$, which gives a threshold in the number of atoms for the existence of the soliton. This is a characteristic of higher-dimensional solitons of cubic nonlinear Schrödinger equations. It is associated with the so-called delocalizing transition [42] [e.g., for values of $N$ smaller than the critical value (minimum of the curve), the solution quickly decays in the uniform background and becomes fully delocalized]. In the right panels of Fig. 1, we also show the corresponding plots in the $E-N$ plane. Notice that, from the general definition $\mu=\frac{d E}{d N}$, the chemical potentials correspond to the slopes of these curves at a given $N$. Also notice that the $E-N$ curves display cusps in correspondence to the turning points of the $\mu-N$ curves.

The stability of the soliton solutions can be inferred from the observed plotted results by means of the VK necessary criterion [43], according to which, stable solutions always correspond to the branches for which $d \mu / d N<0$. We see that no solution can be stable for both attractive and repulsive interactions in the presence of only the NOL (e.g., for $\varepsilon=0$ ). By increasing the strength of the LOL, however, the slope of the curves changes from positive to negative. Therefore, our analytical study predicts that stable solitons in crossed LOLs 

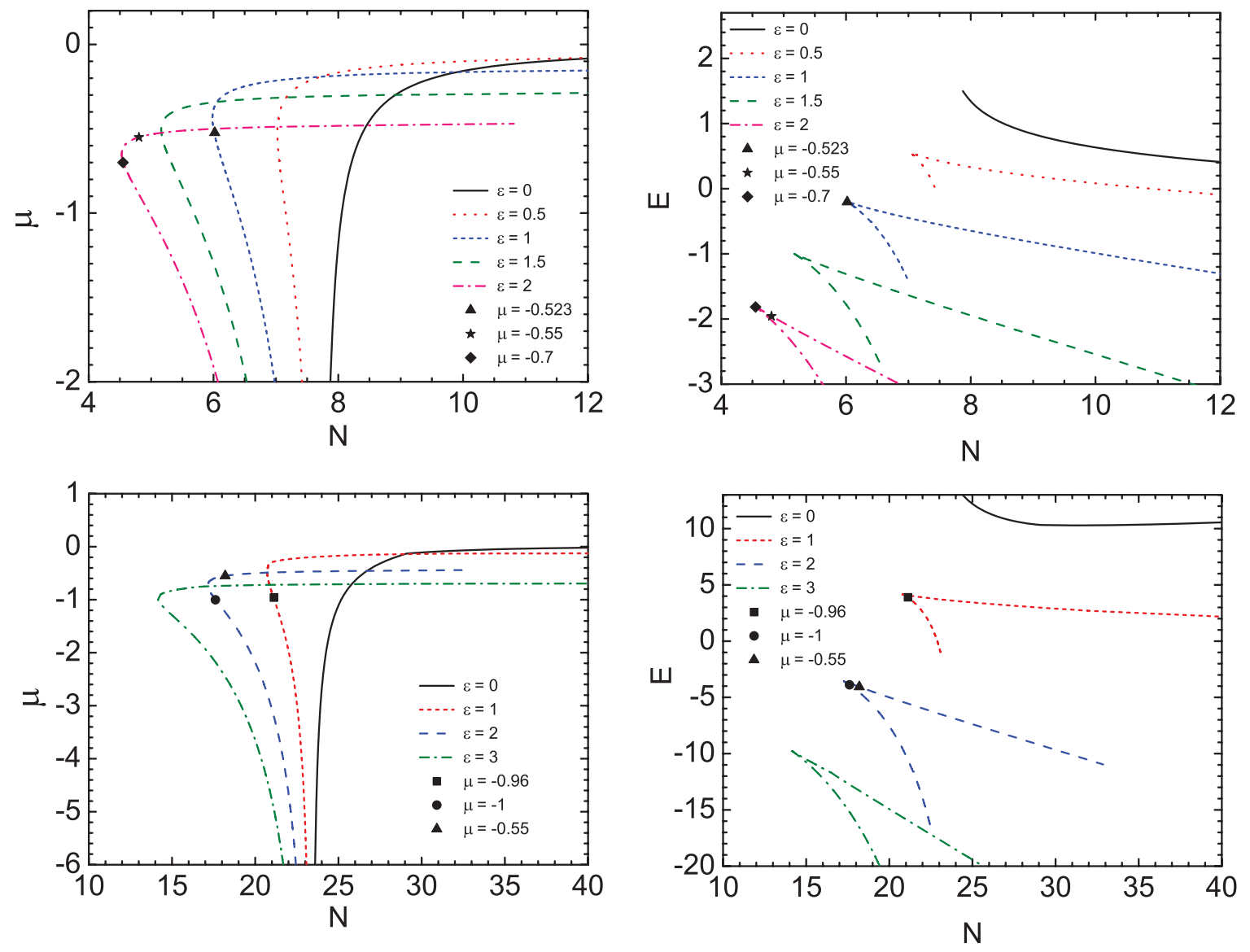

FIG. 2. (Color online) Plots of $2 \mathrm{D}$ solitons in the $\mu-N$ (left panels) and $E-N$ (right panels) planes, as obtained from numerical integrations of Eq. (3), for attractive (upper panels, $\chi=0.5$ ) and repulsive (lower panels, $\chi=-0.5$ ) mean nonlinearities and for increasing strengths $\varepsilon$ of the LOL (see legends inside panels). Other parameters are fixed as in Fig. 1. Symbols on curves correspond to localized solutions selected for comparison with the VA density profiles in Fig. 3 and for stability checks by time propagation in Fig. 4. All the quantities are dimensionless.

and NOLs can exist not only for attractive interactions, a fact that is also true in the absence of the NOL in the $y$ direction as demonstrated in Ref. [23], but also for repulsive interactions (in the absence of the NOL, this last case would be impossible). It is also worth noting that the stable branches of the curve in the attractive case always lie well below the critical threshold for collapse (e.g., $N<N_{\text {cr }}$ ).

In Sec. IV, we show that these predictions are in good agreement with the results obtained by direct numerical integrations of the GPE.

\section{NUMERICAL RESULTS AND VA COMPARISON}

To check the previous analytical predictions, we have performed direct numerical integrations of the 2D GPE in Eq. (3) with crossed LOLs and NOLs. The method we have used to find localized solutions is a standard relaxation algorithm in imaginary time propagation with a back-renormalization, which allows fixing the chemical potential $\mu$ and determining the corresponding number of atoms $N$ [44].

Using this method, we were able to follow these solutions in the parameter space even in the regions where they became unstable (hyperbolic states), which permitted us to trace curves in the $N-\mu$ plane and to determine the existence of the thresholds in the number of atoms (turning points). Typical numerical curves for 2D solitons are depicted in Fig. 2, for both attractive (top panels) and repulsive (bottom panels) interactions for different parameter values. Examples of soliton solutions determined with the relaxation method are shown in the left panels of Fig. 3. To facilitate the comparison with the VA predictions, the same set of parameters as the ones used in Fig. 1 are considered. We see that, in the attractive case, the agreement is very good not only qualitatively, but also from a quantitative point of view. Apart from the small shift of the curves, the VA correctly predicts the existence of the turning point and the change of stability of the solutions. Notice that the agreement improves as the strength of the LOL is increased; this being a consequence of the fact that, in a deeper LOL, the soliton becomes more localized and better described by the Gaussian ansatz used in our variational analysis. For the repulsive case, the agreement becomes more qualitative with a larger shift between the numerical and the VA curves, which is unaffected by the strength of the LOL. This discrepancy can be ascribed to the fact that, for repulsive interactions, the Gaussian ansatz used in the VA becomes less accurate because of the tunneling of the matter into adjacent potential wells (the condensate wave function cannot be localized into a single potential well and develops satellites in adjacent wells of the OL).

In Fig. 3, we compare the sections of the density profiles of the solitons corresponding to the points shown on the $\varepsilon=1.0$ 

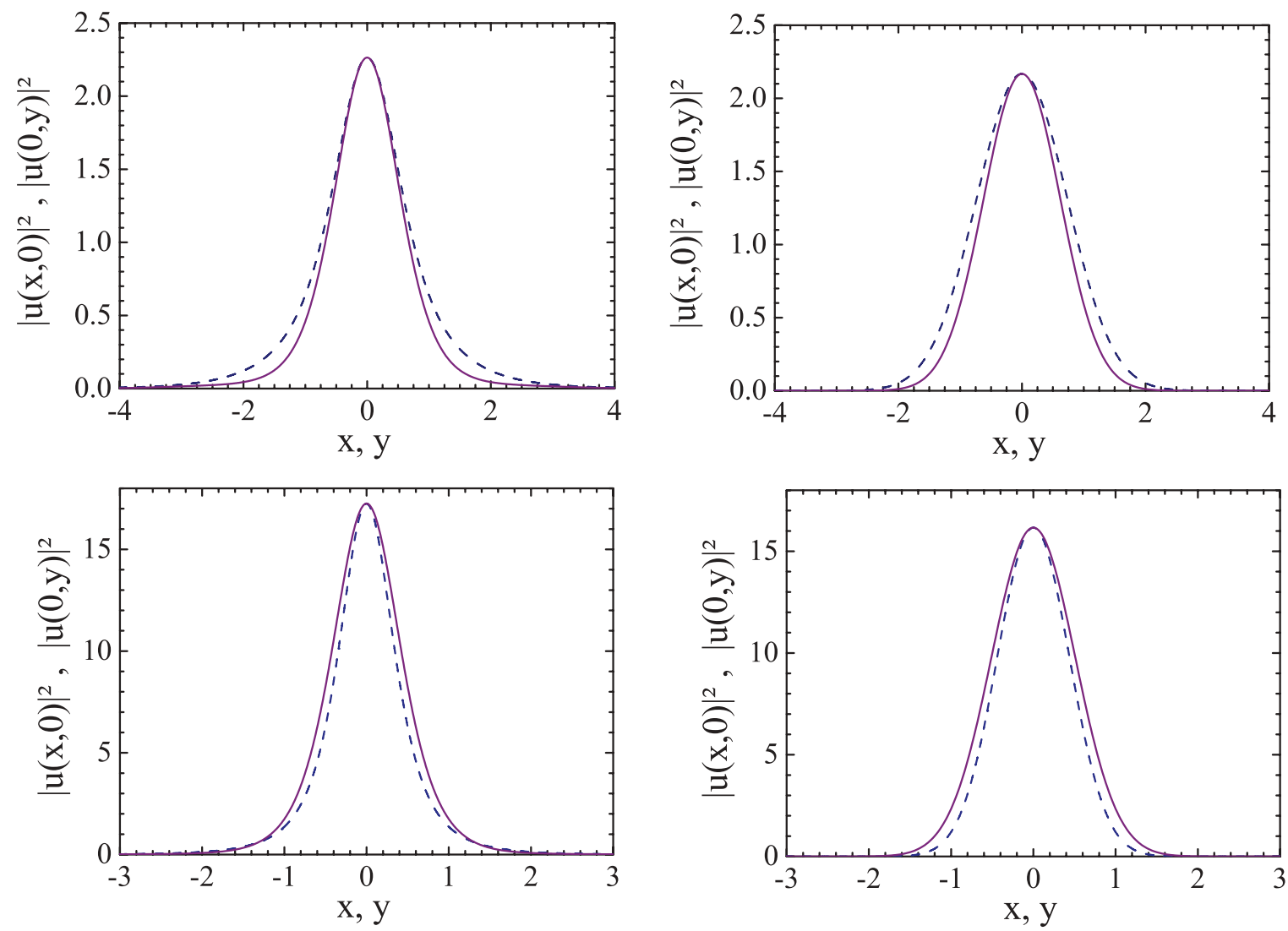

FIG. 3. (Color online) Sections of the soliton density taken at $x=0$ (dashed lines) and $y=0$ (continuous lines) for attractive $\chi=0.5$ (top panels) and repulsive $\chi=-0.5$ (bottom panels) interactions. Left panels refer to the numerical GPE imaginary time relaxation method calculations and correspond to the points shown on the $\varepsilon=1.0$ curves in the upper and lower left panels of Fig. 2, at $N=6.02(\mu=-0.523)$ and $N=21.12(\mu=-0.96)$, respectively. Right panels show the soliton profiles predicted by the VA for the same chemical potentials as in the corresponding left panels. In this case, for $\mu=-0.523$, we have $N=6.2, a=1.3$, and $b=0.928$ (attractive case); and, for $\mu=-0.96$, we have $N=22.69, a=1.93$, and $b=2.6$ (repulsive case). Other parameters are fixed as in Fig. 1. All the quantities are dimensionless.

curves in the corresponding top and bottom left panels of Fig. 2. The right panels refer to VA solutions for attractive (top) and repulsive (bottom) cases, while the corresponding profiles obtained from numerical imaginary time integrations of the GPE are shown in the left panels. We see that, for the chosen parameters, the agreement with our analytical predictions is quite reasonable. Also notice that, for the attractive case (positive mean nonlinearity), the localization induced by the LOL in the $x$ direction is stronger than the one induced by the NOL in the $y$ direction. By changing the sign of the mean nonlinearity, however, keeping all the other parameters the same, the opposite situation occurs.

The stability of these solutions has been checked by means of a real time propagation of the GPE using a Crank-Nicolson split method and taking, as the initial condition, slightly perturbed solitons. In the left panels of Fig. 2, we have selected four specific soliton solutions on branches of the curves, for $\varepsilon=2$, with different slopes close to the turning points, to check stability [e.g., $\mu=-0.7$ (diamond) and $\mu=-0.55$ (star) in the attractive case (top frame); $\mu=-1.0$ (circle) and $\mu=-0.55$ (triangle) in the repulsive case (bottom frame)]. In Fig. 4, we show the dynamics obtained from numerical integrations of the GPE with initial conditions corresponding to these solitons with a small perturbation added. We see that, while the solitons on the branch of the curves with a negative slope are stable under time evolution, the ones on the branches with a positive slope are unstable and quickly decay into a uniform background. These results are in agreement with what is expected from our VA analysis using the VK criterion. From these studies, we conclude that it is possible to create stable 2D BEC solitons using a 1D LOL in the $x$ direction and a periodic modulation of the scattering length in the orthogonal directions, both for attractive and repulsive interactions.

An estimate of the parameters for possible experimental observations of the foregoing 2D solitons can be made by considering, for example, the case of a repulsive BEC with $N \sim 10^{5}$ atoms of ${ }^{87} \mathrm{Rb}$ loaded in a crossed combined OL consisting of an LOL in the $x$ direction with a period $d=2.2 \mu \mathrm{m}$, generated by a laser field of wavelength $\pi / k \approx 0.830 \mu \mathrm{m}$ and strength $\Lambda=1.5 h \mathrm{kHz}$ ( $h=$ the Planck constant), and an NOL in the $y$ direction created by an OFR leading to a spatial variation of the scattering length of the form $a_{s}(y)=a_{s 0}+a_{s 1} \cos (2 \kappa y)$. The background scattering length $a_{s 0}$ is obtained by using a uniform $2 \mathrm{D}$ external magnetic field, with the value near the FR value $B_{\mathrm{FR}}=1007 \mathrm{G}$, which will give $a_{s 0} \approx 100 a_{0}$, with $a_{0}$ denoting the Bohr radius. The modulation part of the scattering length $a_{s 1}$ is induced by illuminating the system in the $y$ direction with a laser field of wavelength $\pi / \kappa \approx 0.784 \mu \mathrm{m}$, corresponding to the 

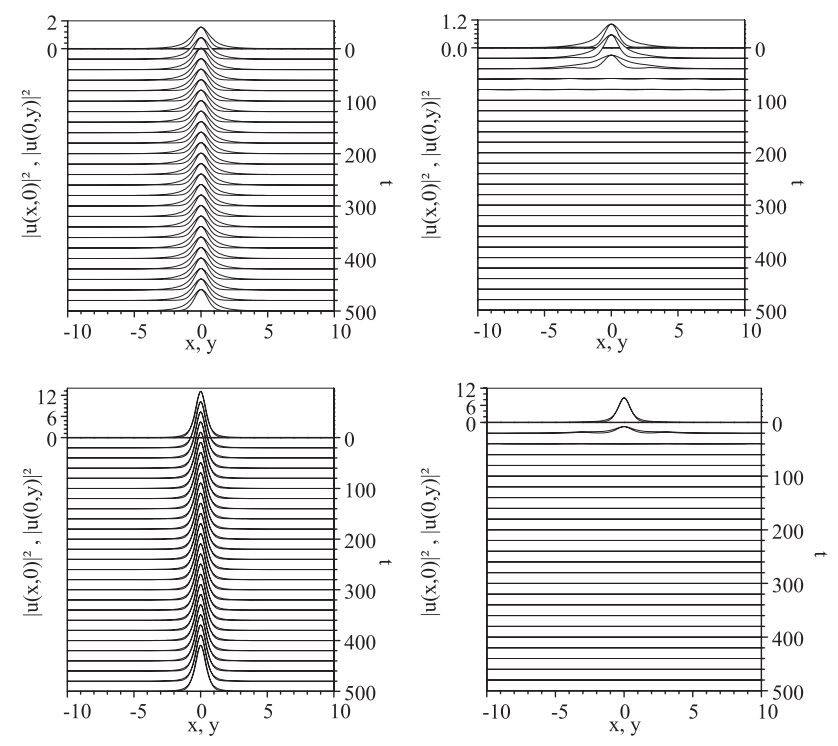

FIG. 4. Time evolution of the $x$ and $y$ sections of the soliton density, stable (left panels) and unstable (right panels), for attractive (top panels) and repulsive (bottom panels) interactions corresponding to the points on the branches of the $\varepsilon=2.0$ curves in the top and bottom panels of Fig. 2. The chemical potentials of the stable and unstable solitons are $\mu=-0.7$ and $\mu=-0.55$ for the attractive case, with $\mu=-1.0$ (left) and $\mu=-0.55$ for the repulsive case, respectively. In the left side of the frames, we show the density scales, with the time evolution shown in the right side. Wider profiles correspond to $y$ sections. All the quantities are dimensionless.

photoassociation wavelength of ${ }^{87} \mathrm{Rb}$, to shift the value of $a_{s 1}$ to values $\operatorname{Re}\left(a_{s 1} / a_{s 0}\right) \approx \pm(1-5)$ [40]. Such a crossed combined lattice is estimated to trap fundamental solitons (e.g., solitons localized in a single potential well) containing up to a few thousand atoms.

\section{CONCLUSIONS}

By summarizing our present findings, we demonstrated that it is possible to stabilize 2D BEC solitons by combining an LOL, in one direction, with an NOL in the other orthogonal direction, where the NOL can be obtained from a periodic modulation of the scattering length. In particular, we have shown that, in 2D crossed LOLs and NOLs, families of 2D solitons can exist and can be stable for both attractive and repulsive interactions. We have determined existent curves for chemical potentials and total energies in terms of the number of atoms, both by a Gaussian variational approach and by direct numerical integrations of the corresponding GPE. As shown, both variational and full-numerical results have been found to be in good agreement. The stability of the solutions has been checked in both cases, by using the VK criterion and by direct numerical real time integrations of the GPE. In this last case, for a few sets of parameters, the time evolutions of density profiles have been presented. These results open the possibility for observing 2D localized matter waves in the presence of crossed LOLs and NOLs in a real experiment, by using, for example, the technique considered in Ref. [40].

\section{ACKNOWLEDGMENTS}

We thank Fundação de Amparo à Pesquisa do Estado de São Paulo (FAPESP) for partial financial support. A.G. and L.T. also acknowledge partial support from Conselho Nacional de Desenvolvimento Científico e Tecnológico (CNPq). M.S. acknowledges FAPESP and the MIUR (PRIN-2008 initiative) for partial financial support. F.K.A. is supported by the Marie Curie program under the Grant No. PIIF-GA-2009236099(NOMATOS). H.L.F.dL. also thanks the Department of Physics "E.R. Caianiello" for the hospitality for a 3-month stay at the University of Salerno.
[1] O. Morsh and M. Oberthaler, Rev. Mod. Phys. 78, 179 (2006).

[2] V. A. Brazhnyi and V. V. Konotop, Mod. Phys. Lett. B 18, 627 (2004).

[3] O. Morsch, J. H. Müller, M. Cristiani, D. Ciampini, and E. Arimondo, Phys. Rev. Lett. 87, 140402 (2001).

[4] I. Carusotto, L. Pitaevskii, S. Stringari, G. Modugno, and M. Inguscio, Phys. Rev. Lett. 95, 093202 (2005).

[5] M. Salerno, V. V. Konotop, and Y. V. Bludov, Phys. Rev. Lett. 101, 030405 (2008).

[6] B. P. Anderson and M. A. Kasevich, Science 282, 1686 (1998).

[7] H. Lignier, C. Sias, D. Ciampini, Y. Singh, A. Zenesini, O. Morsch, and E. Arimondo, Phys. Rev. Lett. 99, 220403 (2007).

[8] Y. V. Bludov, V. V. Konotop, and M. Salerno, Europhys. Lett. 87, 20004 (2009).

[9] A. Zenesini, H. Lignier, D. Ciampini, O. Morsch, and E. Arimondo, Phys. Rev. Lett. 102, 100403 (2009).

[10] M. Jona-Lasinio, O. Morsch, M. Christiani, N. Malossi, J. H. Müller, E. Courtade, M. Anderlini, and E. Arimondo, Phys. Rev. Lett. 91, 230406 (2003); S. Wimberger, R. Mannella,
O. Morsch, E. Arimondo, A. R. Kolovsky, and A. Buchleitner, Phys. Rev. A 72, 063610 (2005).

[11] V. V. Konotop, P. G. Kevrekidis, and M. Salerno, Phys. Rev. A 72, 023611 (2005).

[12] A. Zenesini, H. Lignier, G. Tayebirad, J. Radogostowicz, D. Ciampini, R. Mannella, S. Wimberger, O. Morsch, and E. Arimondo, Phys. Rev. Lett. 103, 090403 (2009).

[13] M. Greiner, O. Mandel, T. Esslinger, T. W. Hänsch, and I. Bloch, Nature (London) 415, 39 (2002).

[14] S. Inouye, M. R. Andrews, J. Stenger, H.-J. Miesner, D. M. Stamper-Kurn, and W. Ketterle, Nature (London) 392, 151 (1998); J. Stenger, S. Inouye, M. R. Andrews, H.-J. Miesner, D. M. Stamper-Kurn, and W. Ketterle, Phys. Rev. Lett. 82, 2422 (1999); J. L. Roberts, N. R. Claussen, J. P. Burke, Jr., C. H. Greene, E. A. Cornell, and C. E. Wieman, ibid. 81, 5109 (1998); S. L. Cornish, N. R. Claussen, J. L. Roberts, E. A. Cornell, and C. E. Wieman, ibid. 85, 1795 (2000); E. A. Donley, N. R. Claussen, S. L. Cornish, J. L. Roberts, E. A. Cornell, and C. E. Wieman, Nature (London) 412, 295 (2001).

[15] A. Trombettoni and A. Smerzi, Phys. Rev. Lett. 86, 2353 (2001).

[16] F. K. Abdullaev, B. B. Baizakov, S. A. Darmanyan, V. V. Konotop, and M. Salerno, Phys. Rev. A 64, 043606 (2001). 
[17] I. Carusotto, D. Embriaco, and G. C. La Rocca, Phys. Rev. A 65, 053611 (2002).

[18] V. V. Konotop and M. Salerno, Phys. Rev. A 65, 021602(R) (2002).

[19] B. Eiermann, T. Anker, M. Albiez, M. Taglieber, P. Treutlein, K.-P. Marzlin, and M. K. Oberthaler, Phys. Rev. Lett. 92, 230401 (2004).

[20] Y. V. Bludov, V. V. Konotop, and M. Salerno, Phys. Rev. A 80, 023623 (2009).

[21] B. B. Baizakov, V. V. Konotop, and M. Salerno, J. Phys. B 35, 5105 (2002); E. A. Ostrovskaya and Y. S. Kivshar, Phys. Rev. Lett. 90, 160407 (2003); J. Yang and Z. Musslimani, Opt. Lett. 28, 2094 (2003).

[22] B. B. Baizakov, B. A. Malomed, and M. Salerno, Europhys. Lett. 63, 642 (2003).

[23] B. B. Baizakov, B. A. Malomed, and M. Salerno, Phys. Rev. A 70, 053613 (2004).

[24] H. Sakaguchi and B. A. Malomed, Phys. Rev. E 72, 046610 (2005); 73, 026601 (2006).

[25] F. K. Abdullaev and J. Garnier, Phys. Rev. A 72, 061605 (2005).

[26] G. Fibich, Y. Sivan, and M. I. Weinstein, Physica D 217, 31 (2006); Y. Sivan, G. Fibich, and M. I. Weinstein, Phys. Rev. Lett. 97, 193902 (2006).

[27] J. Belmonte-Beitia, V. M. Pérez-Garcia, V. Vekslerchik, and P. J. Torres, Phys. Rev. Lett. 98, 064102 (2007).

[28] F. Abdullaev, A. Abdumalikov, and R. Galimzyanov, Phys. Lett. A 367, 149 (2007); Y. V. Kartashov, V. A. Vysloukh, and L. Torner, Opt. Lett. 33, 1747 (2008); H. Sakaguchi and B. A. Malomed, Phys. Rev. A 81, 013624 (2010).
[29] F. K. Abdullaev, A. Gammal, H. L. F. da Luz, and L. Tomio, Phys. Rev. A 76, 043611 (2007).

[30] P. O. Fedichev, Y. Kagan, G. V. Shlyapnikov, and J. T. M. Walraven, Phys. Rev. Lett. 77, 2913 (1996).

[31] R. Yamazaki, S. Taie, S. Sugawa, and Y. Takahashi, Phys. Rev. Lett. 105, 050405 (2010).

[32] G. Dong and B. Hu, Phys. Rev. A 75, 013625 (2007).

[33] F. K. Abdullaev, R. M. Galimzyanov, M. Brtka, and L. Tomio, Phys. Rev. E 79, 056220 (2009).

[34] Y. He, D. Mihalacke, and B. Hu, Opt. Lett. 35, 1716 (2010).

[35] D. S. Petrov and G. V. Shlyapnikov, Phys. Rev. A 64, 012706 (2001).

[36] M. D. Lee, S. A. Morgan, M. J. Davis, and K. Burnett, Phys. Rev. A 65, 043617 (2002).

[37] F. K. Fatemi, K. M. Jones, and P. D. Lett, Phys. Rev. Lett. 85, 4462 (2000).

[38] M. Theis, G. Thalhammer, K. Winkler, M. Hellwig, G. Ruff, R. Grimm, and J. H. Denschlag, Phys. Rev. Lett. 93, 123001 (2004).

[39] G. Thalhammer, M. Theis, K. Winkler, R. Grimm, and J. H. Denschlag, Phys. Rev. A 71, 033403 (2005).

[40] D. M. Bauer, M. Lettner, C. Vo, G. Rempe, and S. Dürr, Nat. Phys. 5, 339 (2009); Phys. Rev. A 79, 062713 (2009).

[41] L. Bergé, Phys. Rep. 303, 259 (1998).

[42] B. B. Baizakov and M. Salerno, Phys. Rev. A 69, 013602 (2004).

[43] N. G. Vakhitov and A. A. Kolokolov, Izv. Vyssh. Uchebn. Zaved., Radiofiz. 16, 1020 (1973) [Radiophys. Quantum Electron. 16, 783 (1973)].

[44] M. Brtka, A. Gammal, and L. Tomio, Phys. Lett. A 359, 339 (2006). 